\title{
Perioperative Management of High Risk Primary Cutaneous Squamous Cell Carcinoma: Role of Radiologic Imaging, Elective Lymph Node Dissection, Sentinel Lymph Node Biopsy, and Adjuvant Radiotherapy
}

\author{
Michael J. Veness • Brent Goedjen • Anokhi Jambusaria
}

Published online: 23 March 2013

(C) Springer Science+Business Media New York 2013

\begin{abstract}
Nonmelanoma skin cancers occur at an epidemic rate in many parts of the world. With an aging population, more patients will develop these cancers and at an ever increasing rate. While in the majority of cases local treatment is curative, a subset of the population will be diagnosed with a high-risk primary cutaneous squamous cell carcinoma. The morbidity and mortality associated with high-risk primary squamous cell carcinoma is usually a consequence of local recurrence and/or the development of metastatic nodal disease. The management of these patients requires knowledge of the role of radiation therapy, elective lymph node dissection, and sentinel lymph node biopsy, which in many cases may be lifesaving, as well as the appropriate imaging modalities to consider in these patients.
\end{abstract}

Keywords Adjuvant radiotherapy · Perineural invasion · Mohs surgery $\cdot$ Squamous cell carcinoma

M. J. Veness

Department of Radiation Oncology, Westmead Hospital, University of Sydney, Westmead,

2145, Sydney, New South Wales, Australia

e-mail: michael.veness@swahs.health.nsw.gov.au

B. Goedjen

Department of Dermatology, Mayo Clinic Florida, 4500 San Pablo Road,

Jacksonville, FL 32216, USA

e-mail: Goedjen.Brent@mayo.edu

A. Jambusaria $(\bowtie)$

Dermatology, Mayo Medical School, Mayo Clinic Florida, 4500 San Pablo Road,

Jacksonville, FL 32224, USA

e-mail: Jambusaria.Anokhi@mayo.edu

\section{Introduction}

With the global population aging, skin cancer is becoming a greater public health concern. Cutaneous squamous cell carcinoma (CSCC) is the second most common skin cancer diagnosed worldwide, with an annual incidence of greater than 750,000 CSCCs [1] in the United States alone. While overall prognosis is excellent, a small subset of tumors, which are usually on the head and neck, recur, metastasize, and cause death [2]. Tumor characteristics associated with poor outcomes include: recurrent tumors, size $>2 \mathrm{~cm}$, depth $(>2 \mathrm{~mm}$ or Clark $>$ IV), anatomic site (ear, lip), poorly differentiated histology, perineural invasion (PNI), or lymphovascular invasion [3-7]. Immunosuppressed patients who develop CSCC or tumors with one or more of the above high-risk pathologic features are referred to as high-risk CSCC patients.

In the setting of high-risk CSCC, the appropriate management decision may be lifesaving. Given these patients are at high risk of recurrent disease either at the primary site or nodal basin, radiologic imaging or sentinel lymph node biopsy may help to identify patients with subclinical disease who could benefit from early and aggressive intervention. Adjuvant radiotherapy has been shown to decrease the risk of local or regional recurrence and may be considered in select high-risk CSCC patients [8]. Unfortunately, the use of these adjuvant modalities has yet to be studied effectively, which has led to a varied approach by clinicians in the utilization of these treatments.

The purpose of this review article was to discuss the management of patients with high-risk primary CSCC and provide evidence based data regarding the role of radiologic imaging and sentinel lymph node biopsy as well as current indications for radiation therapy. As noted earlier, the evidence base to support many recommendations often is weak and limited to single, retrospective, institutional case series 
or systematic review articles. There are no data from randomized, controlled trials.

\section{Incompletely Excised SCC}

Excision with microscopic evaluation of $100 \%$ of surgical margins provides excellent cure rates for high-risk SCC and is the preferred approach [9]. Therefore, clinicians should strive to achieve negative pathologic margins when possible. Incompletely excised SCC often is due to inadequate assessment of pathologic margins or if the tumor is large and complete removal may result in significant morbidity. High-risk CSCC where clear surgical margins cannot be obtained (positive or close margin(s)) remain at risk of poor outcomes. Zitsch et al. reported a worse survival in patients with involved margins compared with patients with clear margins $(P<0.02)$ [10]. When complete surgical resection is not possible, adjuvant radiotherapy may improve outcomes [11]. Babington et al. documented a $37 \%$ local recurrence rate in surgery only patients ( $27 \%$ of which had close/positive margins) vs. a $6 \%$ local recurrence rate in patients treated with surgery and adjuvant radiotherapy (94\% of which had close/positive margins) [12]. In an Australian study of 217 patients with early lower lip SCC (considered as a sun induced cancer), patients treated with excision + local adjuvant radiotherapy had a lower rate of locoregional relapse $(9 \%)$ than those receiving surgery alone (51\%), in the setting of close or positive margins (hazard ratio $0.36 ; 95 \%$ confidence interval $0.17-0.77 ; P=0.0008$ ) [13••]. Therefore, adjuvant radiotherapy should be considered for CSCC if negative pathologic surgical margins cannot be obtained.

\section{Recurrent CSCC}

Patients with local recurrence are at a higher risk of developing regional metastases compared with patients remaining locally controlled. Rowe et al. documented a $32 \%$ incidence of nodal metastases in the setting of recurrent lip cancer [3]. In a large prospective study of 615 German patients with CSCC treated with excision (2-10 $\mathrm{mm}$ excision margins), local recurrence occurred in $3 \%$ of patients $(n=20)$ and of these patients, $30 \%(n=6)$ developed nodal metastases during the 4-year follow-up period [4]. Conversely, in the remaining 595 patients who did not develop a local recurrence, the rate of nodal metastasis was only $3 \%$ $\left(\chi^{2}=25.2, P<0.01\right)$. At a minimum, therefore, clinicians should achieve clear (or negative) excision margins when excising CSCC and consider reexcision surgery or adjuvant radiotherapy (see above) if this is not the case.

\section{Perineural Invasion}

Perineural invasion (PNI) is defined as invasion of tumor cells within the nerve sheath or along the length of the nerve. It is further classified into incidental or microscopic PNI (no clinical or radiographic evidence of PNI, diagnosis is based on histopathologic evidence of tumor cells invading the perineurium) and clinical PNI (positive clinical and/or radiographic evidence of PNI). Approximately $5 \%$ of excised CSCC exhibit incidental PNI and even a smaller minority ( $2 \%$ of all CSCC) will have clinical PNI. Formication (sensation of ants crawling) may herald the diagnosis of PNI [14]. However, dysesthesia, paresthesia, numbness, and pain are all suggestive symptoms. Diagnosis often is delayed, because PNI often is not suspected.

There are no clear guidelines of when to order radiologic imaging to determine the presence and extent of PNI. The NCCN guidelines recommend obtaining a MRI when large nerve involvement is suspected [15]. Even in cases where there is a high-index of suspicion, however, patients can still have normal imaging in the early phase of the disease. Jungehuelsing et al. reported on eight patients with malignant unilateral facial paralysis with initial MRI scans that failed to detect any abnormalities [16].

MRI is considered the imaging modality of choice to detect PNI because of its multiplanar capabilities and better soft tissue definition. It is considered the most sensitive radiographic study to detect and define the extent of PNI [17]. In head and neck tumors, the sensitivity of MRI for detection of PNI was $95 \%$; however, it decreased to $63 \%$ when MRI was tested to outline the entire extent of PNI [18, 19]. Improved technology using 3 Tesla MRI scanners may improve detectability, particularly in early disease when the radiographic changes may be subtle [20]. Typical radiological findings of PNI include: nerve enlargement/enhancement, foraminal enlargement/destruction, obliteration of fat planes and convexity of the lateral cavernous sinus wall [21, 22] (Fig. 1). MRI may be supplemented with a CT scan when there is concern for nerve involvement to the skull base, as CT scans are superior to MRI in outlining bony anatomy. A history of radiation therapy to the site may decrease the accuracy of MRI findings that are consistent with PNI and therefore imaging in post radiation therapy patients should be interpreted accordingly.

Perineural invasion is one of the most common reasons cutaneous oncologists recommend radiotherapy. It is frequently used as an adjuvant modality to improve disease free and overall survival. A large Australian study $(n=1,177)$ of Mohs patients with CSCC documented the presence of PNI in $6 \%$ with over half (53\%) of these patients subsequently receiving adjuvant radiotherapy [23]. In a systematic review involving more than 2,300 patients with high-risk CSCC treated with surgery alone or surgery combined with adjuvant radiotherapy, 
the authors found 91 published cases of CSCC treated with surgery and adjuvant radiotherapy, of which 74 cases had PNI [24].

Radiotherapy has the ability to widely treat at-risk volumes including the involved cranial nerve's pathway, and to include the skull base and intracranial extension back to the brainstem. The utilization of Intensity Modulated Radiotherapy (IMRT) and stereotactic radiotherapy offers a more precise and potentially less toxic treatment $[25,26]$. However, the risk of late central nervous system side effects such as retinopathy and blindness can be serious, and these risks need to be balanced against the potential benefits of treatment and the consequences of recurrent PNI. In addition, the risk of subsequent cutaneous malignancy in the previously irradiated fields must be considered in younger patients.

Since not all tumors with PNI have poor outcomes and radiotherapy has significant risks, there is likely a highestrisk subset of PNI CSCC that warrant adjuvant treatment. To date, this subgroup has yet to be clearly identified. There are several published clinical scenarios where adjuvant radiotherapy may be beneficial. Solid organ transplant recipients and other immunosuppressed patients develop aggressive malignancies and therefore may be an at risk population that should be treated aggressively, even in the setting of microscopic PNI $[27,28]$. Periorbital CSCC with PNI has a high risk of spreading to the orbit, which often is morbid and therefore these tumors may warrant consideration of further treatment [29]. Large caliber nerve invasion may also be a poor prognostic factor. In a retrospective study of $48 \mathrm{CSCC}$ with PNI, Ross et al. found PNI of nerves $\geq 0.1 \mathrm{~mm}$ diameter were significantly associated with nodal metastasis, distant metastasis, and disease-specific death [30]. The finding of extensive PNI of multiple smaller nerves should be considered an indication for adjuvant radiotherapy (indicated in NCCN guidelines), particularly if the primary tumor is recurrent or has a mid-face location in proximity to cranial nerves $\mathrm{V}$ and VII [31•].

PNI tracking down the length of a nerve to the skull base is a unique clinical scenario that may pose a challenge for the treating physician. Adjuvant radiotherapy should be strongly considered in this high-risk subgroup, but surgery may be an option as well. In an Australian study of 21 patients with clinical PNI who underwent surgery (often resecting the Gasserian ganglion), the authors reported no surgical deaths, only three complications, and a 5-year disease-specific survival of $64 \%$. Of note, 18 of 21 patients also received adjuvant radiotherapy [32]. Whether these patients benefit from multimodality treatment is still unclear as opposed to surgery or radiotherapy alone.

Of the published guidelines on the management of CSCC $[15,33-36]$, both the National Comprehensive Cancer Network $(\mathrm{NCCN})$ guidelines [15] and published guidelines in the management of SCC in solid organ transplant patients
[33] recommend consideration of adjuvant radiotherapy for extensive perineural invasion/inflammation or large nerve involvement. Despite these guidelines, there is still variability in the utilization of adjuvant radiotherapy for PNI amongst clinicians. In a recent survey of American Mohs surgeons, most were in favor of recommending a patient for adjuvant radiotherapy in the setting of an involved named branch of a cranial nerve (e.g., temporal branch of the facial nerve) [37]. There was lack of consensus in scenarios not involving a named nerve, with more experienced Mohs surgeons less likely to recommend adjuvant radiotherapy.

The variability in utilization of adjuvant radiotherapy is due to the limited and conflicted data proving its benefit. To date, there are no randomized trials demonstrating an improvement in disease specific or overall survival. The majority of outcome data are based on retrospective institutional case series or systematic reviews, many of which group CSCC with PNI and basal cell carcinoma with PNI and provide outcome data for the group as a whole [24, 38, 39]. These studies have found improved local control and locoregional control rates for incidental PNI (78-87\%) compared to clinical PNI (50-55\%), whether or not adjuvant radiotherapy was employed after surgery. In a systematic review of 91 published cases of CSCC with PNI, there was no difference in outcomes between cases treated with surgery alone $(n=17)$ and those treated with surgery plus adjuvant radiotherapy $(n=74)$ [24]. These studies were subject to treatment bias, in that the treating physician chose to employ adjuvant radiotherapy for an individual patient because he or she thought the patient was at high risk for

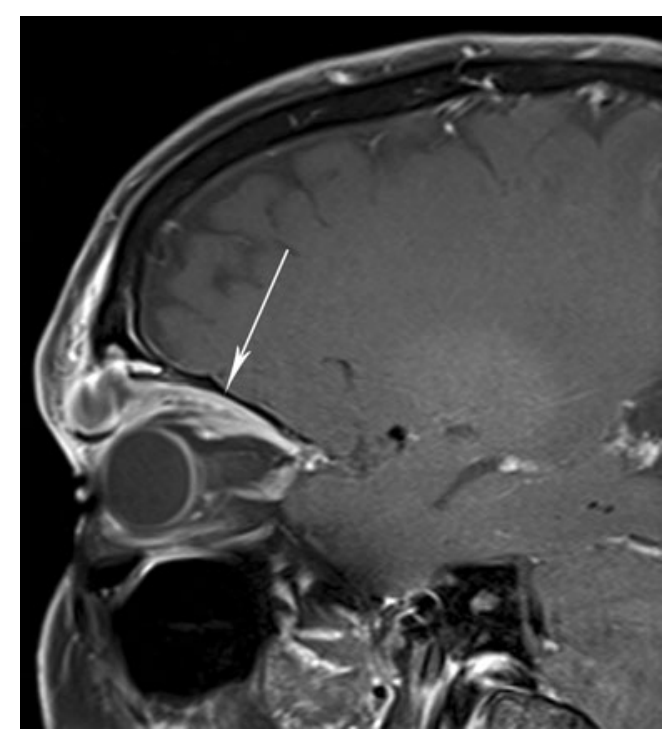

Fig. 1 Sagittal view of a T1-weighted MRI image. There is a heterogeneous, partially cystic mass within the superomedial right orbit extraconal space as well as linear thickening and enhancement of the right supraorbital nerve to the midorbital level consistent with tumor extension of cSCC along the supraorbital nerve (white arrow) 
poor outcomes. Therefore, it is unclear if the lack of survival benefit seen in patients with CSCC with PNI treated with adjuvant radiotherapy is due to lack of effectiveness of the radiation or if these tumors were to have poor outcomes, regardless of treatment. Large, multicenter randomized trials are needed to precisely estimate the benefits of adjuvant radiotherapy, which will ultimately identify clinical scenarios where it should be employed.

\section{Elective Management of Lymph Nodes}

Although only a minority of CSCC patients $(<5 \%)$ will develop metastatic spread to lymph nodes, the incidence may be greater than $10-15 \%$ in a selected high-risk subgroup of patients [40]. Survival following the development of metastatic nodal disease is markedly decreased compared with patients that remain node negative. While the definition of high-risk has been agreed upon [3-7], there is limited data to accurately predict the risk of nodal metastasis $[4,5$, 7], and therefore, it is unclear which risk factors, or combination of risk factors, portend the greatest risk of metastasis. An often quoted tenet is that if the risk of occult regional metastases exceeds $20 \%$, the patient should be considered for elective treatment of regional (first echelon) nodes. As such, clinicians typically account for the number of high risk factors (with higher numbers of risk factors portending greater risk), in conjunction with patient factors and preferences in their decision-making.

Once the decision to electively treat the lymph node basin has been made, clinicians must choose between elective nodal dissection and/or radiotherapy. In an excellent review of the literature, Martinez and Cook highlighted the lack of good quality evidence to guide clinicians on the role of elective neck dissection in patients with high risk CSCC [41]. Yoon et al. [42] reported on 38 patients with external ear CSCC treated predominantly with surgery and noted a $53 \%$ recurrence rate with almost half metastasizing to regional lymph nodes and recommended prophylactic parotidectomy and neck dissection \pm radiotherapy in patients with poor prognostic features, such as cartilage invasion, deep invasion, or poor differentiation. Vartanian et al. suggest patients with T3/T4 lip SCC are at $>20 \%$ risk of having occult spread to upper cervical lymph nodes and should undergo an elective supraomohyoid neck dissection [43]. Breuninger et al. found that desmoplastic CSCC, which is an aggressive histological variant often seen in conjunction with PNI, has a high propensity to regionally metastasize, especially with increasing tumor thickness, and therefore recommended prophylactic nodal dissection in this subgroup of CSCC [44]. However, with a lack of a proven survival benefit with elective nodal dissection, lack of prognostic models that accurately predict risk, the added cost, and potential toxicity with this approach clinicians may alternatively elect a close observation policy. If radiotherapy is chosen, 50 Gy utilizing electron or photon treatment are delivered to the draining lymph node basin over the course of 5 weeks ( 25 daily treatments or fractions) (Fig. 2). Other than acute radiation dermatitis, therapy is generally well tolerated. To date, there are no studies quantifying the benefit of elective radiotherapy in high-risk CSCC in a clinically node-negative patient. In one author's experience (MJV), both modalities have similar control rates, and therefore whether surgery or radiotherapy is recommended needs to be individualized and balanced against the advantages and disadvantages of both treatments. The NCCN guidelines recommend superficial parotidectomy if the CSCC invades to the parotid fascia, which is considered a type of regional metastasis [15].

Given the lack of a clearly defined subgroup of patients who are at highest risk for metastasis, there are no clear indications of when radiologic imaging is warranted to search for nodal metastasis. CT, ultrasound, and ultrasound-guided fine needle aspiration cytology [31•] are among the methods that have been suggested for this purpose, although no optimal approach has been standardized by clinical trials. CT/MRI scans usually add very little to the clinical examination of a node negative region (e.g., cervical neck nodes). Ultrasound is considered by some as the quickest, cheapest, and possibly most sensitive method of evaluating the lymph nodes [4], and some studies suggest it can detect early metastasis more efficiently than palpation, CT, and/or MRI [45]. The published German guidelines for treatment of CSCC recommend ultrasonography of the draining lymph node basin for any tumor $>2 \mathrm{~mm}$ in thickness [35].

\section{Sentinel Lymph Node Biopsy}

An alternative to electively treating nodes at risk is to identify patients with subclinical spread to lymph nodes that

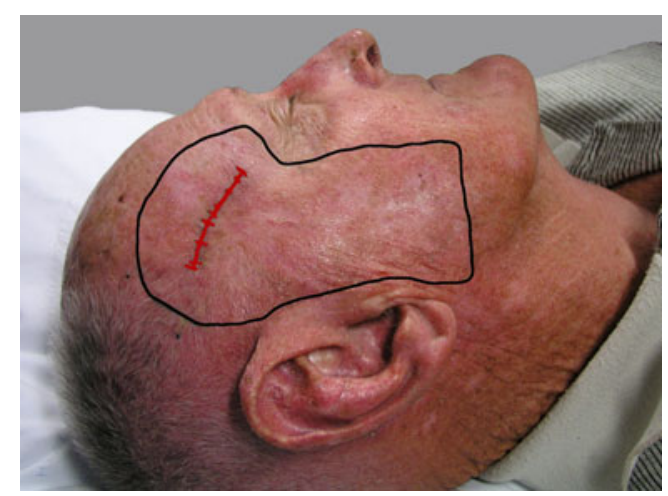

Fig. 2 This patient had a recurrent CSCC which was positive at the deep surgical margin but no evidence of nodal metastasis based on imaging studies. Adjuvant radiotherapy was delivered to the primary site (red line) as well as the preauricular (parotid) nodes. The entire treatment field is outlined in black 
may in turn allow appropriate regional treatment. The concept of sentinel lymph node biopsy (SLNB) has evolved in other malignancies, such as melanoma and breast cancer, to identify patients with occult spread to first echelon lymph nodes, and there is emerging data that SLNB also may have a role in high risk patients with CSCC, although the evidence to support this is relatively sparse [46-49]. Wagner et al. reported on 24 patients with high risk NMSC $(n=17$ with SCC) undergoing SLNB. Seven (29\%) had a positive sentinel node with only one false-positive. The negative predictive value was 0.94 [40]. In another series of nine patients with high-risk CSCC four of nine (44\%) were positive on SLNB with subsequently two dying of metastatic disease. The five with a negative SLNB remained disease free although the median follow-up of 8 months was short [47]. In a recent review of the literature, 130 cases were identified of patients undergoing SLNB for CSCC [49]. The authors report a $10 \%$ rate of a positive SLNB for CSCC located on the head and neck and appropriately suggested that more research is required to further clarify the role of SLNB in CSCC. Identifying patients who would benefit from SLNB in view of the extra surgery and cost is unclear. The NCCN guidelines recommend consideration of sentinel lymph node mapping in certain high-risk lesions but acknowledge that the benefit of this technique has yet to be proven [15].

\section{Metastatic SCC to Lymph Nodes}

Patients with regional metastases from head and neck CSCC are at the highest risk of a poor outcome, because they already have metastatic cancer, as opposed to merely being "at risk" of developing it. Most regional metastases involve head and neck lymph nodes and can be broadly separated into parotid (preauricular and tail of parotid) and cervical (levels I-V) lymph nodes, because these represent the first echelon nodes from primary cutaneous sites on the scalp, forehead, face, lip, ear, and neck [50]. The parotid lymph nodes drain the lymphatic vessels from much of the ipsilateral scalp, temple, forehead, ear, and preauricular face. In Australia, the most frequent parotid malignancy is metastatic CSCC. Although most patients are invariably found to have a past history of skin cancer, approximately $20 \%$ of such patients do not have an obviously identified index lesion that fulfills the criteria for a high-risk CSCC [51]. However, nearly all of these patients will have significant dermatoheliosis on clinical exam.

There is evidence that patients treated with a combined approach incorporating surgery and adjuvant radiotherapy achieve a marked improvement in locoregional control and outcome. In a study of 74 Australian patients with metastatic CSCC to cervical nodes (nonparotid) those undergoing surgery and adjuvant radiotherapy had a lower recurrence rate (15\% vs. $77 \%)$ and significantly better 3-year disease-free survival (70 \% vs. $45 \% ; P=0.02$ ) compared with those treated with surgery alone [52]. In the most recent analysis of the Westmead hospital data of 122 patients with metastatic CSCC to cervical nodes of the head and neck, patients had a markedly improved outcome if they received combined treatment as opposed to surgery alone. Patients who underwent surgery and adjuvant radiotherapy had a 5-year overall survival of $66 \%$ compared with $27 \%$ for patients who underwent surgery alone $(P=0.003)$ [53]. Current best practice for the majority of patients with operable metastatic nodal SCC of the head and neck is surgery and adjuvant radiotherapy.

\section{Conclusions}

Patients with high-risk CSCC are at risk for local recurrence and metastatic disease and employing the appropriate management strategies may decrease morbidity and mortality. Although there is a lack of high-quality data to guide clinicians, any primary tumor with greater than a $20 \%$ risk of developing nodal metastasis should be considered for adjuvant radiotherapy, elective lymph node dissection, or SLNB, as well as further imaging to search for regional metastasis. Clinical scenarios where adjuvant radiotherapy may be beneficial include cases with close/positive surgical margins, recurrent disease, and PNI, but at this time, clinicians will need to use their clinical judgment regarding the indications for its use given the limited available evidence based data. Research is currently being performed to identify these highest-risk tumors, so that these adjuvant treatments can be better studied and appropriate evidence-based guidelines can be developed. Patients with metastatic SCC to the lymph nodes should be promptly referred for both surgery and adjuvant radiotherapy, because multiple studies demonstrate improved survival with combination therapy.

Conflict of Interest M.J. Veness declares no conflicts of interest. B. Goedjen declares no conflicts of interest.

A. Jambusaria declares no conflicts of interest.

\section{References}

Papers of particular interest, published recently, have been highlighted as:

- Of importance

•- Of major importance

1. Rodgers HW, Weinstock MA, Harris AR, et al. Incidence estimate of nonmelanoma skin cancer in the United States. Arch Derm. 2010;146:283-7.

2. Joseph MG, Zulueta WP, Kennedy PJ. Squamous cell carcinoma of the skin. The incidence of metastasis and their outcome. Aust N Z J Surg. 1991;62:697-701. 
3. Rowe DE, Carroll RJ, Day CD. Prognostic factors for local recurrence, metastasis, and survival rates in squamous cell carcinoma of the skin, ear and lip. J Am Acad Dermatol. 1992;26:976-90.

4. Brantsch KD, Meisner C, Schonfisch B, et al. Prospective analysis of the risk factors determining prognosis of cutaneous squamous cell carcinoma. Lancet Oncol. 2008;9:713-20.

5. Clayman GL, Lee JJ, Holsinger FC, et al. Mortality risk from squamous cell skin cancer. J Clin Oncol. 2005;23:59-65.

6. Mullen JT, Feng L, Xing Y, et al. Invasive squamous cell carcinoma of the skin: defining a high-risk group. Ann Surg Oncol. 2006;13:902-9.

7. Jambusaria-Pahlajani A, Kanetsky PA, Karia PS, et al. Evaluation of AJCC Tumor (T) staging for cutaneous squamous cell carcinoma and a proposed alternative tumor staging system. Arch Derm. E-pub (ahead of print).

8. Veness MJ. The important role of radiotherapy in patients with non-melanoma skin cancer and other cutaneous entities. JMIRO. 2008;52:278-86.

9. Pugliano-Mauro M, Goldman G. Mohs surgery is effective for high-risk cutaneous squamous cell carcinoma. Dermatol Surg. 2010;36(10):1544-53.

10. Zitsch RP, Park CW, Renner GJ, Rea JL. Outcome analysis for lip carcinoma. Otolaryngol Head Neck Surg. 1995;113:589-96.

11. Geohas J, Roholt NS, Robinson JK. Adjuvant radiotherapy after excision of cutaneous squamous cell carcinoma. J Am Acad Dermatol. 1994;30:633-6.

12. Babington S, Veness MJ, Cakir B, et al. Squamous cell carcinoma of the lip: is there a role for adjuvant radiotherapy in improving local control following incomplete or inadequate excision? ANZ J Surg. 2003;73:621-5

13. • Najim M, Cross S, Gebski V, et al. Early stage squamous cell carcinoma of the lip: The Australian experience and the benefits of radiotherapy in improving outcome in high-risk patients after resection. Head Neck 2012 [Epub ahead of print]. One of the largest studies to confirm the benefits of adjuvant radiotherapy in reducing the risk of recurrence in high-risk patients.

14. Mendenhall WM, Parsons JT, Mendenhall NP, et al. Carcinoma of the skin of the head and neck with perineural invasion. Head Neck. 1989;11:301-8.

15. Miller SJ. The National Comprehensive Cancer Network (NCCN) guidelines of care for nonemalnoma skin cancers. www.nccn.org.

16. Jungehuelsing $M$, Sittel $C$, Fischbach $R$, et al. Limitations of magnetic resonance imaging in the evaluation of perineural tumor spread causing facial nerve paralysis. Arch Otolaryngol Head Neck Surg. 2000;126:596-601.

17. Nemec SF, Hemeth AM, Czerny C. Perineural tumor spread in malignant head and neck tumors. Top Magn Reson Imaging. 2007;18(6):467-71.

18. Nemzek WR, Hecht S, Gandour-Edwards R, et al. Perineural spread of head and neck tumors: how accurate is MR imaging? AJNR AM J Neuroradiol. 1998;19:701-6.

19. Hanna E, Vual E, Prokopakis E, et al. The sensitivity and specificity of high-resolution imaging in evaluating perineural spread of adenoid cystic carcinoma to the skill base. Arch Otolaryngol Head Neck Surg. 2007;133:541-5.

20. Penn R, Abemayor E, Nabili V, et al. Perineural invasion detected by high-field 3.0-T magnetic resonance imaging. Am J Otolaryngol Head Neck Med Surg. 2010;31:482-4.

21. Galloway TJ, Morris CG, Mancuso AA, et al. Impact of radiographic findings on pronosis for skin carcinoma with perineural invasion. Cancer. 2005; 103:1254-7.

22. Williams LS, Mancuso AA, Mendenhall WM. Perineural spread of cutaneous squamous and basal cell carcinoma: CT and MR detection and its impact on patient management and prognosis. Int J Rad Oncol Biol Phys. 2001;49:1061-9.
23. Leibovitch I, Huilgol SC, Selva D, et al. Cutaneous squamous cell carcinoma treated with Mohs micrographic surgery in Australia. Perineural invasion. J Am Acad Dermatol. 2005;53:261-6.

24. Jambusaria-Pahlajani A, Miller CJ, et al. Surgical monotherapy versus surgery plus adjuvant radiotherapy in high-risk cutaneous squamous cell carcinoma: a systematic review of outcomes. Dermatol Surg. 2009;35:574-85.

25. Gluck I, Ibrahim M, Popovtzer A, et al. Skin cancer of the head and neck with perineural invasion: defining the clinical target volumes based on the patterns of failure. Int J Radiat Oncol Biol Phys. 2009;74:36-46.

26. Fowler BZ, Crocker IR, Johnstone PA. Perineural spread of cutaneous malignancy to the brain; a review of the literature and five patients treated with stereotactic radiotherapy. Cancer. 2005;103:2143-53.

27. Veness MJ, Quinn DI, Ong CS, et al. Aggressive cutaneous malignancies following cardiothoracic transplantation: the Australian experience. Cancer. 1999;85:1758-64.

28. Lott DG, Manz R, Koch C, Lorenz RR. Aggressive behavior of nonmelanotic skin cancers in organ transplant recipients. Transplantation. 2010;90:683-7.

29. Mcnab AA, Francis IC, Benger R, et al. Perineural spread of cutaneous squamous cell carcinoma via the orbit: clinical features and outcome in 21 casts. Opthalmology. 1997;104:1457-62.

30. Ross AS, Whalen FM, Elenitsas RE, et al. Diameter of involved nerves predicts outcome in cutaneous squamous cell carcinoma with PNI: an investigator blinded retrospective cohort study. Derm Surg. 2009;35(12):1859-66.

31. - Mendenhall WM, Ferlito A, Takes RP, et al. Cutaneous head and neck basal and squamous cell carcinomas with perineural invasion. Oral Oncol. 2012;48:918-22. A thorough update on the role of radiotherapy in patients diagnosed with perineural invasion.

32. Panizza B, Solares CA, Redmond M, et al. Surgical resection for clinical perineural invasion from cutaneous squamous cell carcinoma of the head and neck. Head Neck. 2012;34:1622-7.

33. Motley R, Kersey P, Lawrence C, British Association of Dermatologists, British Association of Plastic Surgeons, Royal College of Radiologists, Faculty of Clinical Oncology. Multiprofessional guidelines for the management of the patient with primary cutaneous squamous cell carcinoma. Br J Dermatol. 2002;146(1):18-25.

34. Stasko T, Brown MD, Carucci JA, et al. Guidelines for the management of squamous cell carcinoma in organ transplant recipients. Dermatol Surg. 2004;30(4, pt 2):642-50.

35. Breuninger H, Bootz F, Hauschild A, et al. Short German guidelines: squamous cell carcinoma. J Dtsch Dermatol Ges. 2008;6 suppl 1:S5-8.

36. Committee on Guidelines of Care; Task Force on Cutaneous Squamous Cell Carcinoma. Guidelines for care for squamous cell carcinoma. J Am Acad Dermatol. 1993;28(4):628-31.

37. Jambusaria-Pahlajani A, Hess SD, Katz KA, et al. Uncertainty in the perioperative management of high-risk cutaneous squamous cell carcinoma among Mohs surgeons. Arch Dermatol. 2010;146:1225-32.

38. Han A, Ratner D. What is the role of adjuvant radiotherapy in the treatment of cutaneous squamous cell carcinoma with perineural invasion? Cancer. 2007;109:1053-9.

39. Balamucki CJ, Mancuso AA, Amdur RJ. Skin carcinoma of the head and neck with perineural invasion. Am J Otolaryngol. 2012;33(4):447-54.

40. Cherpelis BS, Marcusen C, Lang PG. Prognostic factors for metastasis in squamous cell carcinoma of the skin. Dermatol Surg. 2002;28:268-73.

41. Martinez JC, Cook JL. High-risk cutaneous squamous cell carcinoma without palpable lymphadenopathy: is there a therapeutic role for elective neck dissection? Dermatol Surg. 2007;3:410-20. 
42. Yoon M, Chougule P, Dufresne R, Wanebo HJ. Localized carcinoma of the external ear is an unrecognized aggressive disease with a high propensity for local regional recurrence. Am J Surg. 1992;164:574-7.

43. Vartanian JG, Carvalho AL, de Araujo Filho MJ, et al. Predictive factors and distribution of lymph node metastasis in lip cancer patients and their implications on the treatment of the neck. Oral Oncol. 2004;40:223-7.

44. Breuninger H, Schaumburg-Lever G, Holzschuh J, Horny HP. Desmoplastic squamous cell carcinoma of skin and vermilion surface. Cancer. 1997;79:915-9.

45. Evans R. Lymph Nodes. In: Ahuja A, Evans R, editors. Practical Head and Neck Ultrasound. London: Greenwich Medical Medial Ltd; 2000. p. 65-82.

46. Reschly MJ, Messina JL, Zaulyanov LL, et al. Utility of sentinel lymphadenectomy in the management of patients with high-risk cutaneous squamous cell carcinoma. Dermatol Surg. 2003;29:135-40.

47. Wagner JD, Evdokimow DZ, Weisberger E, et al. Sentinel node biopsy for high-risk nonmelanoma cutaneous malignancy. Arch Dermatol. 2004;140:75-9.
48. Weisberg NK, Bertagnolli MM, Becker DS. Combined sentinel lymphadenectomy and Mohs surgery for high-risk cutaneous squamous cell carcinoma. J Am Acad Dermatol. 2000;43:483-8.

49. Kwon S, Dong ZM, Wu PC. Sentinel lymph node biopsy for highrisk cutaneous squamous cell carcinoma: clinical experience and review of literature. World J Surg Oncol. 2011;9:80-7.

50. Vauterin TJ, Veness MJ, Morgan GJ, Poulsen MG, O'Brien CJ. Patterns of lymph node spread of cutaneous squamous cell carcinoma of the head and neck. Head Neck. 2006;28:785-91.

51. Veness MJ, Porceddu S, Palme CE, Morgan GJ. Cutaneous head and neck squamous cell carcinoma metastatic to parotid and cervical lymph nodes. Head Neck. 2007;29:621-31.

52. Veness MJ, Palme CE, Smith M, et al. Cutaneous head and neck squamous cell carcinoma metastatic to cervical lymph nodes (nonparotid): a better outcome with surgery and adjuvant radiotherapy. Laryngoscope. 2003;113:1827-33.

53. Wang J, Palme C, Morgan G, et al. Predictors of outcome in patients with metastatic cutaneous head and neck cutaneous squamous cell carcinoma involving cervical lymph nodes: Improved survival with the addition of adjuvant radiotherapy. Head Neck Nov 2011 [Epub a head of print]. 